\title{
Triple Negative Breast Cancer Treatment: Use of Platinum and Platinum Analogs
}

\author{
Manuela Fantini $^{1^{*}}$, Carlotta Santelmo ${ }^{1}$, Fabrizio Drudi ${ }^{{ }^{*}}$, Claudio Ridolfi ${ }^{1}$, Eleonora Barzotti ${ }^{1}$, \\ Lorenzo Gianni ${ }^{1}$, Valentina Arcangeli ${ }^{1}$, Alessandra Affatato ${ }^{1}$, Alberto Ravaioli ${ }^{2}$ \\ ${ }^{1}$ Oncology Department, Infermi Hospital, Rimini, Italy; ${ }^{2}$ IRST (Cancer Center of Romagna), Meldola, Italy. \\ Email: fantamanu77@libero.it, carlotta.santelmo@alice.it, f.drudi1@virgilio.it,claudio.rid@gmail.com, eleonora.barzotti@libero.it, \\ lgianni@auslrn.net, varcangeli@auslrn.net, aaffatato@auslrn.net, aravaioli@auslrn.net
}

Received July 24 $4^{\text {th }}$, 2012; revised August 27 ${ }^{\text {th }}, 2012$; accepted September $9^{\text {th }}, 2012$

\begin{abstract}
Triple negative breast cancer (TNBC) is characterized by a high sensitivity to antiblastic drugs and a high pathological complete remission rate after neoadjuvant therapy. In patients showing complete remission, the probability of developing metastatic disease would seem to be reduced. Nonetheless, this cancer has a high percentage of relapse. Anthracyclines, taxanes and platinum compounds are the most effective drugs for the treatment of TNBC. There is substantial evidence to support the efficacy of platinum-based chemotherapy, probably attributable to the mechanism of action of such drugs, which react with the DNA repair system. PARP inhibitors would also seem to be very interesting. Despite promising results, TNBC remains a disease with a poor prognosis due to the lack of targeted therapy. The discovery of new targets and new agents is thus a high priority issue for this type of breast cancer. In this respect, lipoplatin has been identified as a potentially interesting treatment option to evaluate in both neoadjuvant and advanced settings.
\end{abstract}

Keywords: Breast Cancer; Triple Negative; Treatment; Platinum; Platinum Analogs

\section{Introduction}

Breast cancer is the most common malignancy and leading cause of cancer death in women, both worldwide and in many developed countries, including Italy.

It is a heterogeneous disease whose prognosis is related mainly to stage and biological characterization, which defines a variety of conditions ranging from a low risk of relapse and indolent clinical course to a high relapse risk and rapid clinical progression, despite aggressive therapies [1]. Routine pathological reports, in addition to defining tumor stage and grade, include an assessment of proliferation index (Ki-67/MIB-1 immunostaining), expression of estrogen (ER) and progestin receptors (PR), and HER-2/neu status (evaluated through protein immunostaining or the study of gene amplification). Whilst these biological features have some prognostic value, they are principally considered as predictors of response to different treatments, especially targeted therapies. Breast cancer can be classified into three large subgroups: hormone receptor-positive, HER-2-positive (which is also hormone-receptor positive in less than half of cases), and the so-called "triple negative" (TN) tumors, where ER and PR are negative and there is no amplification or overexpression of HER-2 [2].

*Corresponding authors
Studies of gene expression profiling have confirmed the existence of breast cancer subtypes, leading to a more accurate definition: luminal tumors are typically ERpositive but with different expression of ER-related genes and proliferative genes; HER-2 positive tumors are characterized by the overexpression and/or amplification of HER-2 and other genes; the normal breast-like subtype and basal-like subtype are usually TN at immunostaining studies [3]. This classification has biological and clinical relevance, defining how tumor growth is driven by different pathways, as best exemplified by the luminal and HER2/neu subtypes, and with different prognoses. TN tumors merit special attention because they have a poor prognosis and also because no targeted therapy has been approved as yet for clinical use [4]. TN tumors defined by immunostaining differ somewhat from the basal-like subtype defined by gene profiling, but the latter subtype is the most frequent and most important form of triple negative breast cancer (TNBC) [5]. The most important features of TN/basal-like tumors include a strong expression of basal cytokeratins CK5/6 and CK17 (high molecular weight cytokeratins, found in the basal layer of stratified epithelia), expression of proliferation related genes, high tumor grade, frequent p53 mutations and p63 gene amplification, low expression of BRCA1, frequent expression of EGFR, KIT, vimentin, p- 
cadherin, fascin, caveolins 1 and 2, alpha-B-crystallin, and genes involved in matrix remodeling and angiogenesis.

Using univariate analysis, we can define the differences between the two main histological subgroups. In Bertucci and co-workers 2008 study on TNBC, the median age of patients with basal tumors (50 years) was lower than that of patients with other TN types (57 years). Basal tumors exhibited a higher pathological grade: $88 \%$ were grade III versus only $51 \%$ in non basal tumors. The pathological tumor size (pT) was higher in basal tumors: $60 \%$ pT2 - pT3 versus $46 \%$ in the other group. No correlation was found with pathological axillary lymph node status $(\mathrm{pN})$. There were more medullary breast cancers within the basal tumors, and more lobular breast cancers within the non basal tumors. Five-year metastasis-free survival was $65 \%$ in the basal group and $75 \%$ in the other group, while 5-year overall survival (OS) was $68 \%$ and $78 \%$, respectively. Pathological complete response (pCR) after primary chemotherapy was observed in 59\% of basal tumors versus $27 \%$ in the other group [6].

TNBC represents a heterogeneous group of tumors that include $70 \%$ of basal tumors and $30 \%$ of the non basal kind. TN basal tumors are characterized by ER, PR and HER-2 negativity, and EGFR and cytokeratin 5/6 and 17 positivity. In contrast, TN non basal tumors are characterized by ER, PR and HER-2 negativity and EGFR and cytokeratin 5/6 and 17 negativity. Nevertheless, it is impossible to distinguish between basal and non basal subtypes in the results obtained from the clinical treatment of TN tumors in neoadjuvant, adjuvant and metastatic settings because they are not considered separately in either clinical practice or in the majority of published papers.

\section{Treatment of TNBC}

TNBC is characterized by a high sensitivity to antiblastic drugs. Although an elevated percentage of pCR is observed in the neoadjuvant setting and a high remission rate in metastatic disease, this breast cancer subtype shows a high percentage of relapse. Numerous drugs have been evaluated for the treatment of this tumor, the most efficacious being anthracyclines, taxanes (paclitaxel and docetaxel) and platinum compounds. Among the new drugs, PARP-inhibitors would seem to be of particular interest. However, because of the biological heterogeneity within TNBC, many patients show an excellent outcome while others obtain much poorer results, despite the administration of systemic treatments considered effective.

\subsection{The Experience in Neoadjuvant Settings}

In the neoadjuvant treatment of TNBC, the paradox of high sensitivity to anthracyclines and a high percentage of relapse in this poor prognostic subgroup is attributable to the elevated recurrence rate in patients who have residual disease at the time of surgery. Those who achieve pCR in basal-like, HER-2 positive and ER negative tumors would seem to show a higher rate of survival, in contrast to patients who do not achieve pCR and appear more likely to experience early relapse and early death. One of the most important studies published to date is by Carey and coworkers who examined the response rate to neoadjuvant anthracycline-based chemotherapy in $34 \mathrm{TN}$ patients, reporting $85 \%$ clinical response (complete or partial), more than the value reached in luminal subtypes [7]. pCR to chemotherapy was significantly better among basal-like subtypes (27\%) than among other subtypes (7\%). Furthermore, the percentage of patients with minimal residual disease after chemotherapy was higher in basal-like (58\%) than in luminal A subtypes (21\%). Despite sensitivity of basal like-TNBC in Carey's study, his group still showed very poor prognosis, for the high relapse rates observed among those who do not achieve pCR.

In another study by Liedtke and coworkers, response to neoadjuvant chemotherapy and survival was compared in TNBC and non-TNBC patients [8]. 1118 patients at M.D. Anderson Cancer Center received neoadjuvant chemotherapy for stage I-III breast cancer from 1985 to 2004. Although patients with TNBC (23\%) had higher pCR rates than those with non-TNBC (22\% versus $11 \%)$, they showed poorer 3-year progression-free (PFS) and overall survival ( $p<0.0001$ and $p<0.0001$, respectively). Recurrence and death rates were higher for the TNBC group, but only in the first three years. When pCR was achieved, patients with TNBC or non-TNBC showed similar survival while those with residual disease had a poorer outcome, especially if they had the TNBC subtype, confirming previous observations in larger groups of patients.

\subsection{The Experience in Adjuvant Settings}

Numerous studies have reported on the adjuvant treatment of TNBC basal-like and non basal-like TNBC, the majority concerning retrospective experiences analyzing breast cancer treatments in different subsets. Although locoregional and distant relapse in TNBC occur mainly during the first three years of follow up after the end of therapy, we found few reports on long-term follow up that made a distinction between TN and other kinds of breast cancers. In Voduc and coworkers's report of 4033 patients followed up for almost ten years after breastconserving surgery and given post-operative radiotherapy and hormone or antiblastic treatment, regional relapsefree survival (RRFS) was $86 \%$ for basal-like breast cancer and $92 \%$ for non basal-like TNBC, while the percentage of RRFS of luminal A breast cancer was 97\% [1]. The observation that TNBCs are especially sensitive to 
antiblastic drugs has been made by numerous authors. The Cancer and Leukemia Group B reported a benefit from the addition of a taxane to the adjuvant treatment of ER, PR and HER-2 negative tumors, whereas no benefit was observed in patients with ER positive tumors [9]. Furthermore, the Breast Cancer Intergroup, in their review of 6644 node-positive breast cancer patients, reported more important results in those with ER- negative tumor given chemotherapy [9]. Patients with a high proliferative index and ER/PR negativity showed very impressive results at ten years with CMF chemotherapy. More recently, Colleoni and his collaborators published a paper on the use of classic CMF in TN node-negative breast cancer patients [10]. They found that CMF chemotherapy produced a stronger effect in patients with TNBC than in those who did not receive the schedule or whose tumors showed ER positivity.

New approaches to adjuvant therapy are guided by results from preclinical models, molecular profiling, and findings from trials on metastatic disease. Numerous adjuvant clinical trials are currently ongoing to evaluate the role of ixabepilone, platinum agents and VEGF in this clinical setting [11]. The PACS 08 adjuvant trial [12], built on data from PACS 01 [13,14], is a phase III study of TNBC patients randomized to receive three cycles of FEC followed by three cycles of docetaxel or ixabepilone every three weeks. The phase III adjuvant TITAN trial is evaluating four cycles of standard doxorubicin plus cyclophosphamide followed by four cycles of ixabepilone or twelve cycles of weekly paclitaxel [15]. The phase III BEATRICE study is assessing numerous chemotherapy agents with or without bevacizumab at a dosage of 5 $\mathrm{mg} / \mathrm{Kg}$ weekly for patients with TNBC [16]. Findings are expected in the next year.

In conclusion, TN tumors are currently most effecttively treated with conventional adjuvant or neoadjuvant therapies (anthracyclines and taxanes). Although significant results have been achieved, TN patients show lower disease-free survival than those with non $\mathrm{TN}$ tumors.

\subsection{The Experience in a Metastatic Setting}

Results on the treatment of TNBC in a metastatic setting do not differ from those described in neoadjuvant and adjuvant situations. These tumors only respond to chemotherapy, and although a substantial percentage of complete response (CR) can be achieved, relapse and non clinical response remain high. Whilst anthracyclines and taxanes have proven the most effective drugs in this setting, cisplatin also shows very promising results [17]. Among the new products, PARP-inhibitors are considered to be potentially important drugs. Indeed some reports suggest that TNBC expresses high levels of polymerase 1 (PARP-1), a DNA repair enzyme which is the molecular target of PARP inhibitors. In a randomized phase II study in patients with metastatic TN disease, the PARP inhibitor BSI-201 significantly improved objective response rates ( $48 \%$ vs $16 \%, \mathrm{p}=0.002$ ), PFS (6.9 vs 3.3 months, $\mathrm{p}<0.0001)$ and median OS (9.2 vs 5.7 months, $\mathrm{p}=0.0005$ ) when added to carboplatin and gemcitabine [11]. However, despite such encouraging findings, prognosis in TNBC patients with advanced disease (apart from those with accurately verified $\mathrm{CR}$ ), remains very poor.

\subsection{Platinum and Lipoplatinum Experience}

Although cisplatin is one of the oldest and more effective anticancer drugs, its use may be limited by adverse events, mainly nephrotoxicity and neurotoxicity.

A number of studies evaluating platinum agents in patients with metastatic TNBC have been carried out. A retrospective study by Uhm and coworkers comparing outcome in patients with TNBC with that of patients with other BC subtypes, all treated with a taxane-platinum regimen, did not highlight a difference in response rates between these two groups [18]. A similar experience from the UK showed that patients with advanced TNBC treated with platinum-based chemotherapy had ORRs similar to those with non-TN tumors [17].

Favorable results have also been obtained with cisplatin in a metastatic setting. A single institution phase II study on weekly cisplatin and metronomic cyclophosphamide reported that such a regimen appeared to be safe and effective, with an ORR of $63 \%$ and median time to progression of 13 months in contrast to $33 \%$ and 7 months, respectively, in patients not treated with cisplatin $[19,20]$. The association of carboplatin with paclitaxel [21] or irinotecan [22] in pretreated metastatic patients with $\mathrm{TN}$ tumours has also shown good response rates (57\% and $30 \%$, respectively). A study conducted at the Royal Marsden Institute also supported the hypothesis that patients with TN tumors have a significantly longer PFS compared with that of other histological subtypes, with a trend towards an improved OS when treated with platinum.

Very few studies have been conducted on lipoplatinum in advanced TNBC. Only recently Farhat and coworkers reported the results of their phase II trial of lipoplatin and vinorelbine combination in patients with HER-2 negative metastatic breast cancer who had not received prior treatment in an advanced setting [23]. A 53.1\% ORR was reported (CR in 3 patients, 9.4\%) and 37.5\% patients showed stable disease. The majority of adverse events were mild or moderate. For this reason lipoplatin would seem to be an attractive treatment option for these patients.

Lipoplatin is a novel liposomal formulation of cisplatin, designed to increase safety and tolerability by decreasing toxicity and nephrotoxicity. It decreases the 
exposure of organs to cisplatin, whilst effectively delivering the drug to the tumor. The formulation comprises cisplatin and liposomes based on 1,2 dipalmitoyl-snglycero-3-phospho-rac-1-glycerol (sodium salt) (DPPG, mw 745), soy phosphatidyl choline (SPC-3, mw 790), cholesterol (CHOL, mw 386.66) and methoxy-polyethylene glycol-distearoyl phosphatidyl-etha-nolamine lipid conjugate (mMPEG2000-DSPE, mw 2807). At steady state, particles have a weight ratio of $8.9 \%$ cisplatin to 91.1\% lipids (0.9:9.1). The total lipid to cisplatin ratio in lipoplatin is $10 \mathrm{mg}$ lipid/mg cisplatin. The cholesterol content of lipoplatin is $11.6 \%$ of the total lipids. It is calculated that $1.3 \mathrm{mg}$ of cholesterol is injected per $\mathrm{mg}$ of cisplatin [24]. There are numerous indications that point towards the potential usefulness of lipoplatin as treatment of TNBC. Consequently, our group is currently planning a new trial on patients with TNBC who have become resistant to anthracyclines and taxanes, offering lipoplatin as second-line treatment following progression after firstline therapy.

\section{Conclusion}

TNBC is a very serious illness and conventional treatments only obtain partial control of the disease, and normally for a short period of time. The search for new treatments is urgently needed and lipoplatin could be potentially useful in advanced disease and could also represent a new possible treatment in the future in neoadjuvant and adjuvant settings.

\section{REFERENCES}

[1] K. D. Voduc, M. C. U. Cheang, S. Tyldesley, et al., "Breast Cancer Subtypes and the Risck of Local and Regional Relapse,” Journal of Clinical Oncology, Vol. 28, No. 10, 2010, pp. 1684-1691. doi:10.1200/JCO.2009.24.9284

[2] T. O. Nielsen, F. D. Hsu, K. Jensen, et al., "Immunohistochemical and Clinical Characterization of the Basal like Subtype of Invasive Breast Carcinoma," Clinical Cancer Research, Vol. 10, No. 16, 2004, pp. 5367-5374. doi:10.1158/1078-0432.CCR-04-0220

[3] L. J. Van’t Veer, H. Dai, M. J. Van De Vijver, et al., "Gene Expression Profiling Predicts Clinical Outcome of Breast Cancer," Nature, Vol. 415, No. 6871, 2002, pp. 530-536. doi:10.1038/415530a

[4] R. Dent, M. Trudeau, K. L. Pritchard, et al., "Triple Negative Breast Cancer: Clinical Features and Patterns of Reccurence,” Clinical Cancer Research, Vol. 13, No. 15, 2007, pp. 4429-4434.

[5] M. C. Cheang, D. Voduc, C. Bajdik, et al., "Basal like Breast Cancer Defined by Five Biomarkers Has Superior Prognostic Value than Triple-Negative Phenotype," Clinical Cancer Research, Vol. 14, No. 15, 2008, pp. 13681376. doi:10.1158/1078-0432.CCR-07-1658

[6] F. Bertucci, P. Finetti, N. Cervera, et al., "How Basal
Are Triple Negative Breast Cancers?” International Journal of Cancer, Vol. 123, No. 1, 2008, pp. 236-240. doi:10.1002/ijc.23518

[7] L. A. Carey, E. C. Dees, L. Sawyer, et al., "Triple Negative Paradox: Primary Tumor Chemosensitivity of Breast Cancer Subtypes," Clinical Cancer Research, Vol. 13, No. 8, 2007, pp. 2329-2334. doi:10.1158/1078-0432.CCR-06-1109

[8] C. Liedtke, C. Mazouni, K. R. Hess, et al., "Response to Neoadjuvant Therapy and Long-Term Survival in Patients with Triple-Negative Breast Cancer,” Journal of Clinical Oncology, Vol. 26, No. 8, 2008, pp. 1275-1281. doi:10.1200/JCO.2007.14.4147

[9] D. A. Berry, C. Cirrincione, I. C. Henderson, et al., "Estrogen-Receptor Status and Outcomes of Modern Chemotherapy for Patients with Node-Positive Breast Cancer," The Journal of the American Medical Association, Vol. 295, No. 14, 2006, pp. 1658-1667. Erratum in: JAMA, Vol. 295, No. 20, 2006, p. 2356. doi:10.1001/jama.295.14.1658

[10] M. Colleoni, B. F. Cole, G. Viale, et al., "Classical Cyclofosphamide, Methotrexate, and Fluorouracil Chemotherapy Is More Effective in Triple Negative, NodeNegative Breast Cancer: Results from Two Randomized Trials of Adjuvant Chemotherapy for Node Negative Breast Cancer,” Journal of Clinical Oncology, Vol. 28, No. 18, 2010, pp. 2966-2973. doi:10.1200/JCO.2009.25.9549

[11] A. Perez, A. Moreno-Aspitia, E. Aubrey Thompson, et al., "Adjuvant Therapy of Triple Negative Breast Cancer," Breast Cancer Research and Treatment, Vol. 120, No. 2, 2010, pp. 285-291. doi:10.1007/s10549-010-0736-z

[12] M. Campone, “NCT00630032 (PACS 08) Randomized, Open Label, Multicentric Phase III Evaluating the Benefit of a Sequential Regimen Associating FEC 100 and Ixapilone in Adjuvant Treatment of Non Metastatic, Poor Prognosis Breast Cancer Defined as Triple Negative Tumor [HER2 Negative-ER Negative-PR Negative] or [HER2 Negative and PR Negative] Tumor in Node Positive or Node Negative Patients,” 2009.

http://clinicaltrials.gov

[13] H. S. Rugo, H. Roche, E. Thomas, et al., "Ixabepilone plus Capecitabine vs Capecitabine in Patients with Triple Negative Tumors: A Pool Analysis of Patients from Two Large Phase III Clinical Studies,” Cancer Research, Vol. 69, No. 2, 2009, p. 3057. doi:10.1158/0008-5472.SABCS-3057

[14] J. Baselga, V. Semiglazov, P. Van Dam, et al., "Phase II Randomized Study of Neoadjuvant Everolimus Plus Letrozole Compared with Placebo in Patients with Estrogen Receptor-Positive Breast Cancer," Journal of Clinical Oncology, Vol. 27, No. 16, 2009, pp. 2630-2637. doi:10.1200/JCO.2008.18.8391

[15] D. A. Yardley, "NCT00789581 (Titan) Phase III Study of Doxorubicin/Cyclophosphamide (AC) Followed by Ixabepilone vs AC Followed by Paclitaxel in Patients with Triple-Negative Early-Stage Breast Cancer,” 2011. http://clinicaltrials.gov 
[16] Hoffmann-La Roche, "NCT00528567 (BEATRICE) an Open Label 2-Arm Study to Evaluate the Impact of Adjuvant Bevacizumab on Invasive Disease Free Survival in Triple Negative Breast Cancer,” 2012. http://clinicaltrials.gov

[17] B. Sirohi, M. Arnedos, S. Popat, et al., "Platinum-Based Chemotherapy in Triple Negative Breast Cancer,” Annals of Oncology, Vol. 19, No. 11, 2008, pp. 1846-1852. doi:10.1093/annonc/mdn395

[18] J. E. Uhm, Y. H. Park, S. Y. Yi, et al., "Treatment Outcomes and Clinicopathologic Carachteristics of Triple Negative Breast Cancer Patients Who Received Platinum-Containing Chemotherapy," International Journal of Cancer, Vol. 124, No. 6, 2009, pp. 1457-1462. doi:10.1002/ijc. 24090

[19] G. S. Bhattacharyya, S. Basu, V. Agarwal, et al., "Single Institution Phase II Study of Weekly Cisplatinum and Metronomic Dosing of Cyclophosphamide and Methotrexate in Second Line Metastatic Breast Cancer Triple Negative,” European Journal of Cancer, Vol. 18, Supp. 7, 2009, (Abstract 41).

[20] C. Oakman, G. Viale, A. Di Leo, et al., "Management of
Triple Negative Breast Cancer,” The Breast, Vol. 19, No. 5, 2010, pp. 312-321. doi:10.1016/j.breast.2010.03.026

[21] J. W. Chia, P. Ang, H. See, et al., "Triple Negative Metastatic/Recurrent Breast Cancer: Treatment with Paclitaxel/Carboplatin Combination Chemotherapy,” Journal of Clinical Oncology, Vol. 25, Supp. 18, 2007, pp. 1086.

[22] J. O’Shaughnessy, D. Weckstein, S. Vukelja, et al., "Preliminary Results of a Randomized Phase II Study of Weekly Irinotecan/Carboplatin with or without Cetuximab in Patients with Metastatic Breast Cancer,” 2007 San Antonio Breast Cancer Symposium, San Antonio, December 2007, [Abstract 308].

[23] F. Farhat, S. Temraz, J. Kattan, et al., "Preliminary Results of a Phase II Study of Lipoplatin (Liposomal Cisplatin)/Vinorelbine Combination as First Line Treatment in HER2/Neu Negative Metastatic Breast Cancer (MBC)," Clinical Breast Cancer, Vol. 11, No. 6, 2011, pp. 384389. doi:10.1016/j.clbc.2011.08.005

[24] R. Terkola, “Liposomal Cisplatin: Lipoplatin,” European Journal of Oncological Pharmacy, Vol. 1, No. 2, 2007, pp. 15-20. 